\title{
AN INTEGRABILITY THEOREM FOR POWER SERIES
}

\author{
S. M. MAZHAR
}

1. Concerning integrability of a power series we prove the following theorem:

Theorem. Let $\lambda(t)$ be a positive function defined on $(0,1]$ such that $\lambda(t) t^{-\delta}$ is non-increasing for some $\delta>0$ and

$$
\sum_{n=k}^{\infty} \lambda(1 / n) n^{-2} \leq M \lambda(1 / k) k^{-1} .
$$

Let $f(x)=\Sigma_{0}^{\infty} c_{n} x^{n}, 0 \leq x<1$. Suppose $\left\{\rho_{n}\right\}$ is a positive increasing sequence with $\Sigma_{1}^{\infty} \frac{1}{n \rho_{n}}<\infty$ such that

$$
c_{n}>\frac{-K}{\left(\rho_{n} \lambda(1 / n)\right)^{1 / p} n^{1-1 / p}}, 0<p<\infty, K>0
$$

for all sufficiently large $n$. Then $\lambda(1-x)|f(x)|^{p} \in L(0,1)$ iff

$$
\sum_{n=1}^{\infty} \lambda(1 / n) n^{-2}\left(\sum_{k=0}^{n}\left|c_{k}\right|\right)^{p}<\infty .
$$

This generalizes a theorem of Leindler [4], where he assumes that $\lambda(t)$ is a nonincreasing function. Also our theorem enables us to deduce a theorem of Jain [1] for all $\gamma<1$ by choosing $\lambda(t)=t^{-\gamma}$ with $\delta>-\gamma$, whereas Leindler's theorem includes her theorem only for $0 \leq \gamma<1$.

2. We need the following lemma for the proof of our theorem.

Lemma. Let $\lambda(t)$ be a positive function such that $\lambda(t) \in L(0,1), \lambda(t) t^{-\delta}$ is nonincreasing for some $\delta>0$ and $\lambda\left(\frac{1}{n+1}\right)=O(\lambda(1 / n))$. Let $F(x)=\Sigma_{0}^{\infty} a_{k} x^{k}, 0 \leq x<$ $1, a_{k} \geq 0$ then for $0<p \leq \infty$

$$
\begin{aligned}
\lambda(1-x) F^{p}(x) & \in L(0,1) \text { iff } \\
\sum_{n=1}^{\infty} \lambda(1 / n) n^{-2} S_{n}^{p} & <\infty \text { where } S_{n}=\sum_{k=0}^{n} a_{k} .
\end{aligned}
$$

Received Appil 23, 1990.

Presently on sabbatical leave from Kuwait University, Box 5969, 13060-Safat-KUWAIT. 
This generalizes a previous theorem of Leindler [3].

Proof of the lemma. The proof is similar to that of Khan [2] or Leindler [3]. Since $(1-1 / n)^{n}$ is increasing we have for $\frac{1}{n+1} \leq y \leq \frac{1}{n}, n \geq 2$

$$
F(1-y) \geq \sum_{0}^{n} a_{k}(1-y)^{k} \geq \sum_{k=0}^{n} a_{k}\left(1-\frac{1}{n}\right)^{n} \geq \frac{S_{n}}{4}
$$

Hence

$$
\begin{aligned}
\sum_{n=1}^{\infty} \lambda\left(\frac{1}{n}\right) n^{-2} S_{n}^{p} & \leq 2^{\delta+1} \sum_{n=1}^{\infty} \int_{1 / n+1}^{1 / n} \lambda(y) y^{-\delta} y^{\delta} S_{n}^{p} d y \\
& =O(1)+O\left(\sum_{n=2}^{\infty} \int_{1 / n+1}^{1 / n} \lambda(y)(F(1-y))^{p} d y\right) \\
& =O\left(\int_{0}^{1} \lambda(1-x) F^{p}(x) d x\right)+O(1) .
\end{aligned}
$$

Thus $(2.1) \Rightarrow(2.2)$.

To prove the converse we have

$$
\begin{aligned}
& \int_{0}^{1} \lambda(1-x) F^{p}(x) d x=\sum_{n=1}^{\infty} \int_{1 / n+1}^{1 / n} \lambda(y)\left(\sum_{k=0}^{\infty} a_{k}(1-y)^{k}\right)^{p} d y \\
\leq & \sum_{n=1}^{\infty} \int_{1 / n+1}^{1 / n} \lambda(y)\left(\sum_{k=0}^{\infty} a_{k}\left(1-\frac{1}{n+1}\right)^{k}\right)^{p} d y \\
\leq & \sum_{n=1}^{\infty} \lambda\left(\frac{1}{n+1}\right)\left(\frac{n+1}{n}\right)^{\delta}\left(\sum_{k=0}^{\infty} a_{k}\left(1-\frac{1}{n+1}\right)^{k}\right)^{p} \frac{1}{n(n+1)} \\
= & O\left(\sum_{n=1}^{\infty} \lambda\left(\frac{1}{n}\right) n^{-2}\left(\sum_{k=0}^{\infty} a_{k}\left(1-\frac{1}{n+1}\right)^{k}\right)^{p}\right)
\end{aligned}
$$

since $\lambda\left(\frac{1}{k+1}\right)=O(\lambda(1 / k))$ which follows in view of $(1.1)$ and the fact that

$$
\sum_{n=k}^{\infty} \lambda\left(\frac{1}{n}\right) n^{-2} \geq \lambda\left(\frac{1}{k+1}\right)(k+1)^{\delta} \sum_{n=k+1}^{\infty} n^{-2-\delta} \geq \frac{1}{1+\delta}(1+k)^{-1} \lambda\left(\frac{1}{k+1}\right) .
$$

Suppose $p \geq 1$, then proceeding as in [4], we have

$$
\int_{0}^{1} \lambda(1-x) F^{p}(x) d x=O(1) \sum_{n=1}^{\infty} \lambda\left(\frac{1}{n}\right) n^{-2} \sum_{i=1}^{\infty} 2^{-\frac{i p}{2}} S_{n i}^{p}
$$




$$
\begin{aligned}
& =O(1) \sum_{i=1}^{\infty} 2^{-\frac{i p}{2}} \sum_{n=1}^{\infty} \lambda\left(\frac{1}{n}\right) n^{-2} S_{n i}^{p}=O(1) \sum_{i=1}^{\infty} 2^{-\frac{i p}{2} i^{2+\delta}} \sum_{n=1}^{\infty} \lambda\left(\frac{1}{n i}\right)(n i)^{-2} S_{n i}^{p} \\
& =O(1) \sum_{i=1}^{\infty} i^{2+\delta} 2^{-\frac{i p}{2}} \sum_{m=1}^{\infty} \lambda\left(\frac{1}{m}\right) m^{-2} S_{m}^{p}=O(1) \sum_{m=1}^{\infty} \lambda\left(\frac{1}{m}\right) m^{-2} S_{m}^{p},
\end{aligned}
$$

since $\sum_{i=1}^{\infty} 2^{-\frac{i p}{2}} i^{2+\delta}<\infty$.

Now suppose $0<p<1$. Then

$$
\begin{aligned}
& \sum_{k=0}^{\infty} a_{k}\left(1-\frac{1}{n+1}\right)^{k} \leq S_{n}+\sum_{k=n}^{\infty} e^{-\frac{k}{n+1}} a_{k} \\
& \leq S_{n}+\sum_{j=1}^{\infty} \sum_{k=j n}^{(j+1) n-1} a_{k} e^{-\frac{k}{n+1}} \leq S_{n}+\sum_{j=1}^{\infty} e^{-\frac{j n}{n+1}} \sum_{k=j n}^{(j+1) n-1} a_{k} \\
& \leq S_{n}+K \sum_{j=1}^{\infty} e^{-j / 2} S_{(j+1) n} .
\end{aligned}
$$

Hence from (2.3)

$$
\begin{aligned}
& \int_{0}^{1} \lambda(1-x) F^{p}(x) p d x \\
= & O\left(\sum_{n=1}^{\infty} \lambda\left(\frac{1}{n}\right) n^{-2} S_{n}^{p}\right)+O\left(\sum_{n=1}^{\infty} \lambda\left(\frac{1}{n}\right) n^{-2} \sum_{j=1}^{\infty} e^{\left.-\frac{p j}{2} S_{(j+1) n}^{p}\right)}\right. \\
= & O\left(\sum_{n=1}^{\infty} \lambda\left(\frac{1}{n}\right) n^{-2} S_{n}^{p}\right)+O\left(\sum_{j=1}^{\infty} e^{\left.-\frac{p j}{2} \sum_{n=1}^{\infty} \lambda\left(\frac{1}{n}\right) n^{-2} S_{(j+1) n}^{p}\right)}\right. \\
= & O\left(\sum_{n=1}^{\infty} \lambda\left(\frac{1}{n}\right) n^{-2} S_{n}^{p}\right)+O\left(\sum_{i=2}^{\infty} e^{\left.-\frac{p(i-1)}{2} i^{2+\delta} \sum_{m=1}^{\infty} \lambda\left(\frac{1}{m}\right) m^{-2} S_{m}^{p}\right)}\right. \\
= & O\left(\sum_{m=1}^{\infty} \lambda\left(\frac{1}{m}\right) m^{-2} S_{m}^{p}\right) .
\end{aligned}
$$

Thus $(2.2) \Rightarrow(2.1)$.

3. Proof of the theorem. Let $A(x)=\Sigma_{0}^{\infty} b_{n} x^{n}, 0 \leq x<1$ with

$$
b_{n}=\frac{K}{\left(\rho_{n} \lambda(1 / n)\right)^{1 / p} n^{1-\frac{1}{p}}}, n \geq 1
$$


Using the inequality [4]

$$
\sum_{n=1}^{\infty} \lambda_{n}\left(\sum_{k=1}^{n} b_{k}\right)^{p} \leq p^{p} \sum_{n=1}^{\infty} \lambda_{n}^{1-p}\left(\sum_{k=n}^{\infty} \lambda_{k}\right)^{p} b_{n}^{p}
$$

which holds for any $\lambda_{n}>0, b_{n} \geq 0, p \geq 1$, we have with $\lambda_{n}=\lambda(1 / n) n^{-2}$ and in view of (1.1)

$$
\begin{aligned}
& \sum_{n=1}^{\infty} \lambda(1 / n) n^{-2}\left(\sum_{k=1}^{n} b_{k}\right)^{p} \leq O(1) \sum_{1}^{\infty}\left(\lambda\left(\frac{1}{n}\right) n^{-2}\right)^{1-p}\left(\lambda\left(\frac{1}{n}\right) n^{-1}\right)^{p} b_{n}^{p} \\
= & O\left(\sum_{1}^{\infty} \lambda\left(\frac{1}{n}\right) n^{-2+p} b_{n}^{p}\right)=O\left(\sum_{1}^{\infty} \lambda\left(\frac{1}{n}\right) n^{-2+p} \frac{1}{\rho_{n} \lambda\left(\frac{1}{n}\right) n^{p-1}}\right)=O\left(\sum_{n=1}^{\infty} \frac{1}{n \rho_{n}}\right)=O(1) .
\end{aligned}
$$

If $0<p<1$, then

$$
\begin{aligned}
\sum_{n=2}^{\infty} \lambda\left(\frac{1}{n}\right) n^{-2}\left(\sum_{k=1}^{n} b_{k}\right)^{p} & \leq \sum_{m=1}^{\infty} \sum_{n=2^{m}+1}^{2^{m+1}} \lambda\left(\frac{1}{n}\right) n^{-2}\left(\sum_{k=3}^{2^{m+1}} b_{k}\right)^{p}+O(1) \\
& \leq 2^{\delta} \sum_{m=1}^{\infty} \lambda\left(\frac{1}{2^{m+1}}\right) 2^{-m}\left(\sum_{k=3}^{2^{m+1}} b_{k}\right)^{p}+O(1) .
\end{aligned}
$$

Now

$$
\begin{gathered}
\sum_{k=3}^{2^{m+1}} b_{k}=K \sum_{k=1}^{m} \sum_{n=2^{k}+1}^{2^{k+1}} \frac{n^{\frac{1}{p}-1+\frac{\delta}{p}}}{\left(\rho_{n} \lambda\left(\frac{1}{n}\right) n^{\delta}\right)^{1 / p}} \\
\leq K \sum_{k=1}^{m} \frac{2^{(k+1)\left(\frac{1}{p}-1+\frac{\delta}{p}\right)} 2^{k}}{\left(\rho_{2^{k}} \lambda\left(\frac{1}{2^{k}}\right) 2^{k \delta}\right)^{1 / p}} \leq K \sum_{k=1}^{m} \frac{2^{k / p}}{\left(\rho_{2^{k}} \lambda\left(\frac{1}{2^{k}}\right)\right)^{1 / p}} .
\end{gathered}
$$


Hence

$$
\begin{aligned}
& \sum_{n=2}^{\infty} \lambda\left(\frac{1}{n}\right) n^{-2}\left(\sum_{k=1}^{n} b_{k}\right)^{p} \\
\leq & K \sum_{m=1}^{\infty} \lambda\left(\frac{1}{2^{m+1}}\right) 2^{-m} \sum_{k=1}^{m} \frac{2^{k}}{\rho_{2^{k}} \lambda\left(\frac{1}{2^{k}}\right)}+O(1) \\
= & K \sum_{k=1}^{m} \frac{2^{k}}{\rho_{2^{k}} \lambda\left(\frac{1}{2^{k}}\right)} \sum_{m=k}^{\infty} 2^{-m} \lambda\left(\frac{1}{2^{m+1}}\right)+O(1) \\
\leq & K \sum_{k=1}^{\infty} \frac{2^{k}}{\rho_{2^{k}} \lambda\left(1 / 2^{k}\right)} \lambda\left(1 / 2^{k}\right) 2^{-k}+O(1) \\
= & K \sum_{k=1}^{\infty} \frac{1}{\rho_{2^{k}}}+O(1) \\
\leq & K \sum_{n=1}^{\infty} \frac{1}{n \rho_{n}}+O(1) \\
= & O(1),
\end{aligned}
$$

since

$$
\begin{aligned}
\sum_{n=k}^{\infty} 2^{-n} \lambda\left(\frac{1}{2^{n}}\right) & \leq 2^{2+\delta} \sum_{n=k}^{\infty} \sum_{m=2^{n}+1}^{2^{n+1}} m^{-2} \lambda\left(\frac{1}{m}\right) \\
& =2^{2+\delta} \sum_{m=2^{k}+1}^{\infty} m^{-2} \lambda\left(\frac{1}{m}\right) \leq K \lambda\left(\frac{1}{2^{k}}\right) 2^{-k}
\end{aligned}
$$

by (1.1).

Thus for $0<p<\infty$ the condition (2.2) of the lemma is satisfied. Hence

$$
\lambda(1-x)(A(x))^{p} \in L(0,1) .
$$

Now proceeding exactly as in [4] we complete the proof of the theorem.

\section{References}

1. P. Jain, "An integrability theorem for power series," Publ. Math. Debrecen 20 (1973), 129-131.

2. R. S. Khan, "On power series with positive coefficients," Acta Sci. Math. 30(1969), 255-257.

3. L. Leindler, "Note on power series with positive coefficents," Acta. Sci. Math. 30(1969), $259-261$.

4. L. Leindler, "An integrability theorem for power series," Acta Sci. Math. 38(1976), 103-105. 